\title{
The mass transfer research in complex porous media and pipelines by spectral methods
}

\author{
Pyanylo Ya., Sobko V., Bratash O. \\ Centre of Mathematical Modelling of Pidstryhach Institute \\ for Applied Problems of Mechanics and Mathematics \\ National Academy of Sciences of Ukraine \\ 15 Dudayev str., 79005, Lviv, Ukraine
}

(Received 22 December 2017)

\begin{abstract}
The method of solving problems of mathematical physics, in particular for pressure distribution finding in the water in the underground gas storage layers on the basis of the biorthogonal polynomials constructed by the authors is proposed in the paper. The way of the problem solving by the method of separation of variables on the basis of the biorthogonal polynomials is studied. The solution of the problem is found in the form of the series sum of the biorthogonal and quasi-spectral polynomials. The comparative analysis for the different values of parameters is performed. The impact of the methods parameters, in particular the partial sum order, the bit grid and the calculation error on the accuracy of the solution obtained is studied. The calculation results are presented in the form of the tables. The algorithm of the process of the gas motion in the pipelines using fractional derivatives is constructed.
\end{abstract}

Keywords: spectral methods, mathematical model, pressure distribution, orthogonal polynomials, biorthogonal polynomials, quasi-orthogonal polynomials, partial differential equations, fractional derivatives equations.

2000 MSC: $12 \mathrm{E} 10$

UDC: $519.6: 539.3$

DOI: $10.23939 / \mathrm{mmc} 2017.02 .187$

\section{Introduction}

The setting of the parameters of the underground gas storage work for the gas taking off (pumping) process keeping in the presence of the water in the layers is an important problem for their effective exploitation. The water presence is found practically in all of the gas storages (gas deposits) created in the depleted deposits. Nowadays, with a large number of researches an exhaustive theory for describing of the processes occurring in the layers-collectors of the gas storages in the presence of water does not exist yet. Due to the increase in the cost of the energy carriers the requirements for the calculation of the underground gas storages work parameters increase. Since, there is the water in the underground gas storages layers the part of the gas can diffuse or dissolve in it. The proportion of the gas present in the water largely depends on the pressure distribution in it. It requires the formation of appropriate adaptive models and methods which using the measured parameters (pressure, flow, etc.), allow to determine the pressure distribution in the water, that will give an opportunity to estimate the amount of the gas in it. To solve this problem the spectral methods are used in this paper.

The spectral methods are used both in theoretical studies and for solving a wide class of problems of mathematics and mechanics. Their essence is that the functions included into the model are presented in the form of orthogonal series in accordance to the selected basis. The solution finding is reduced to the coefficients calculating of the orthogonal series of the desired solution. It is shown $[1,2]$ that the choice of the orthogonal basis should be coordinated with the definition domain of the desired solution. The positive sides are those that many orthogonal bases are studied well enough, they are easy to use, and the solving algorithms constructed on their basis are easy for automation. The negative side is 
that the summing of corresponding series is, as a rule, an incorrect problem. Further not all the criteria that are set to the solutions of the problems can be satisfied by means of using of one orthogonal basis. Due to this fact, to meet the broader criteria we modify the existing bases or construct new ones. One of the methods of these comments consideration is the use of the biorthogonal expansions. Nowadays, there are a few papers devoted to their research and practical application. It is mainly due to the fact that the formation of these biorthogonal bases is related to significant difficulties of calculation and they are not studied enough.

\section{Formulation of the problem}

An infinite layer of the thickness $l, 0<y<l$ is considered. The pressure values on the boundaries are equal to $\varphi_{1}(t)$ and $\varphi_{2}(t)$. The initial pressure is $f(x)$. The calculation of the water pressure distribution $p(y, t)$ in a flat infinite medium of the thickness $l$ is determined as the solution of onedimensional filtration equation $[3,4]$

$$
\frac{\partial}{\partial y}\left[\frac{k h}{\mu}\left(\frac{\partial p}{\partial y}+\rho g\right)\right]=2 \alpha m h \frac{\partial p}{\partial t},
$$

where $k$ is the layer permeability, $\mu$ is the dynamic viscosity of the water, $\alpha$ is the water saturation coefficient, $m$ is the porosity of the layer. Since the layer thickness is insignificant and is considered to be constant, the equation (1) has the form

$$
\frac{\partial}{\partial y}\left(\frac{k}{\mu} \frac{\partial p}{\partial y}\right)=2 \alpha m \frac{\partial p}{\partial t} .
$$

In this case, the problem is as follows:

find the solution of the equation

$$
\varkappa \frac{\partial^{2} p}{\partial y^{2}}=\frac{\partial p}{\partial t} \quad(0<y<l)
$$

under the initial condition

$$
p(y, 0)=f(y),
$$

and the boundary conditions

$$
p(0, t)=\varphi_{1}(t), \quad p(l, t)=\varphi_{2}(t) .
$$

As the boundary conditions we will consider the following: on the bottom surface of the layer, the pressure can be considered to be constant, equal to hydrostatic pressure of the water $p_{n}$; on the upper surface, the pressure value is calculated on the basis of the hydraulic coupling GCP - obliteration zone - GWC [1-3] and is also considered to be constant $p_{v}$.

The initial distribution of the water pressure in the water layer

$$
p(y, 0)=\rho g\left(h_{0}+y\right), \quad 0<y<l .
$$

The solution of the problem is a partial solution of a more general problem. Here $\varkappa=k /(2 \mu \alpha m)$.

\section{Solving the problem}

Since the boundless layer of the thickness $l, 0<y<l$, is considered and functions which help us to solve this problem are considered on the interval $[-1,1]$, then we make a replacement $y=0.5 l(x+1)$. Then

$$
p(y, t)=p\left(\frac{l(x+1)}{2}, t\right)=P(x, t),
$$




$$
\frac{\partial^{2} p(y, t)}{\partial y^{2}}=\frac{4}{l^{2}} \frac{\partial^{2} P(x, t)}{\partial x^{2}}
$$

Considering the formula (5), we write the equation (2) and the conditions (3), (4) in the form

$$
\begin{gathered}
\varkappa \frac{4}{l^{2}} \frac{\partial^{2} P(x, t)}{\partial x^{2}}=\frac{\partial P(x, t)}{\partial t}, \\
P(x, 0)=\rho g \frac{l(x+1)}{2}, \\
P(-1, t)=\varphi_{1}=\text { const, } \quad P(1, t)=\varphi_{2}=\text { const. }
\end{gathered}
$$

The solution of the problem (6)-(8) we find in the form $[5,6]$

$$
P(x, t)=\sum_{i=1}^{n+2} V_{i}^{n+\bar{i}}(x) G_{i}(t),
$$

where $V(x)$ are the basis functions, for which the following relations are true

$$
\begin{aligned}
& \frac{d^{2} V_{i}^{n+\bar{i}}(x)}{d x^{2}}=-\frac{V_{i}^{n+\bar{i}}(x)}{\lambda_{i+(-1)^{n}+1}^{n}}+\frac{\tau_{i+(-1)^{\bar{i}+1}}^{n}}{\lambda_{i+(-1)^{\bar{i}+1}}^{n}} T_{n+1+\bar{i}}^{\prime}(x), \quad i=1, \ldots, n, \\
& \frac{d^{2} V_{n+1}^{n+1}(x)}{d x^{2}}=\frac{(n+2)^{2} \pi}{8} \sum_{k=1}^{\frac{n}{2}} \frac{\bar{c}_{2}^{2 k}}{\lambda_{2 k}^{n}} \frac{V_{2 k-1}^{n+1}(x)}{N_{2 k-1}^{n}}+\frac{1}{15} n(n+1)(n+3)(n+4) T_{n+2}^{\prime}(x), \\
& \frac{d^{2} V_{n+2}^{n}(x)}{d x^{2}}=\frac{(n+1)^{2} \pi}{2} \sum_{k=1}^{\frac{n}{2}} \frac{\bar{c}_{1}^{2 k-1}}{\lambda_{2 k-1}^{n}} \frac{V_{2 k}^{n}(x)}{N_{2 k}^{n}}+\frac{1}{15}(n-1) n(n+2)(n+3) T_{n+1}^{\prime}(x),
\end{aligned}
$$

where $V_{n+1}^{n+1}(x)=T_{n+2}^{\prime}(x), V_{n+2}^{n}(x)=T_{n+1}^{\prime}(x), \bar{i}=0$ for the even values of $i$, and $\bar{i}=1$ for the odd values of $i$. $T_{n+1}=T_{n+1}(x)$ and $T_{n+2}=T_{n+2}(x)$ are the Chebyshev polynomials of degree $n+1$ and $n+2, \lambda_{i}^{n}, i=1, \ldots, n$ are the eigenvalues of the integral operator $\pi_{1}^{\infty} L=\pi_{1}^{\infty} \int_{-1}^{x} \int_{-1}^{x_{1}}$, $U_{2 i}^{2 s}(x)=\sum_{j=1}^{s} c_{2 j}^{2 i} \tilde{T}_{2 j}(x), U_{2 i-1}^{2 s-1}(x)=\sum_{j=1}^{s} c_{2 j-1}^{2 i-1} \tilde{T}_{2 j-1}(x)$ are the eigenfunctions of this operator, $\tilde{T}_{j}(x)$ are modified Chebyshev polinomials, $\bar{U}_{2 i}^{2 s}(x)=\sum_{j=1}^{s} \bar{c}_{2 j}^{2 i} T_{2 j}(x), \bar{U}_{2 i-1}^{2 s-1}(x)=\sum_{j=1}^{s} \bar{c}_{2 j-1}^{2 i-1} T_{2 j-1}(x)$ are the eigenfunctions of this turned operator, $\tau_{2 i}^{2 s}(x)=\frac{c_{2 s}^{2 i}}{4(2 s+1)(2 s+2)}, \tau_{2 i-1}^{2 s}(x)=\frac{c_{2 s-1}^{2 i-1}}{4(2 s)(2 s+1)}, \bar{\tau}_{2 i}^{2 s}(x)=$ $\frac{\bar{c}_{2 s}^{2 i}}{4(2 s)(2 s+1)}, \bar{\tau}_{2 i-1}^{2 s}(x)=\frac{\bar{c}_{2 s-1}^{2 i-1}}{4(2 s-1)(2 s)}$, are the biorthogonal functions $V_{2 i-\bar{i}}^{n+\bar{i}}(x)=\int_{-1}^{x} U_{2 i-1+\bar{i}}^{n-\bar{i}-(-1)^{\bar{i}}}\left(x_{1}\right) d x_{1}$, $\bar{V}_{2 i-\bar{i}}^{n+\bar{i}}(x)=-\sqrt{1-x^{2}} \int_{-1}^{x} \frac{\bar{U}_{2 i-1+\bar{i}}^{n-\bar{i}-(-1)^{\bar{i}}}\left(x_{1}\right)}{\sqrt{1-x_{1}^{2}}} d x_{1}, i=1, \ldots, n / 2, N_{i}^{n}=\int_{-1}^{1} \frac{V_{i}^{n+\bar{i}}(x) \bar{V}_{i}^{n+\bar{i}}(x)}{\sqrt{1-x^{2}}} d x, i=1, \ldots, n$ is the norm of the biorthogonal functions $[5,6]$. Let us substitute (9) into the equation (6) and obtain

$$
\varkappa \frac{4}{l^{2}} \sum_{i=1}^{n+2} \frac{\partial^{2} V_{i}^{n+\bar{i}}(x)}{\partial x^{2}} G_{i}(t)=\sum_{i=1}^{n+2} V_{i}^{n+\bar{i}}(x) \frac{\partial G_{i}(t)}{\partial t} .
$$

Let us multiply the equation (10) by $V_{i}^{n+\bar{i}}(x), i=1, \ldots, n$ and integrate it with respect to $x$ with the weight $r(x)=\left(1-x^{2}\right)^{-1 / 2}$ from $x=-1$ to $x=1$. We will obtain the system of $n$ differential equations of the first order of the kind

$$
\frac{4}{l^{2}}\left(-\frac{N_{i}}{\lambda_{i+(-1)^{\bar{i}+1}}^{n}} G_{i}(t)+\frac{(n+1+\bar{i})^{2}}{2^{2 \bar{i}+1}} \pi \frac{\bar{c}_{1+\bar{i}}^{i+(-1)^{\bar{i}+1}}}{\lambda_{i+(-1)^{\bar{i}+1}}^{n}} G_{n+2-\bar{i}}(t)\right)=N_{i} \frac{\partial G_{i}(t)}{\partial t}, \quad i=1, \ldots, n .
$$


From the boundary conditions (7), considering $V_{i}(-1)=V_{i}(1)=0$, we have

$$
\frac{\varphi_{1}+\varphi_{2}}{2(n+1)^{2}}=G_{n+2}(t), \quad \frac{\varphi_{2}-\varphi_{1}}{2(n+2)^{2}}=G_{n+1}(t) .
$$

Taking into account the formula (12), we write the system (11) in the form

$$
\frac{\partial G_{i}(t)}{\partial t}+\frac{4 \varkappa}{l^{2} \lambda_{i+(-1)^{\bar{i}+1}}^{n}} G_{i}(t)=\frac{\varkappa \pi}{2^{2 \bar{i}} l^{2} N_{i}} \frac{\bar{c}_{1+\bar{i}}^{i+(-1)^{\bar{i}+1}}}{\lambda_{i+(-1)^{\bar{i}+1}}^{n}}\left(\varphi_{2}+(-1)^{i} \varphi_{1}\right), \quad i=1, \ldots, n .
$$

Therefore, the following functions will be the solutions of the equations of the system (13)

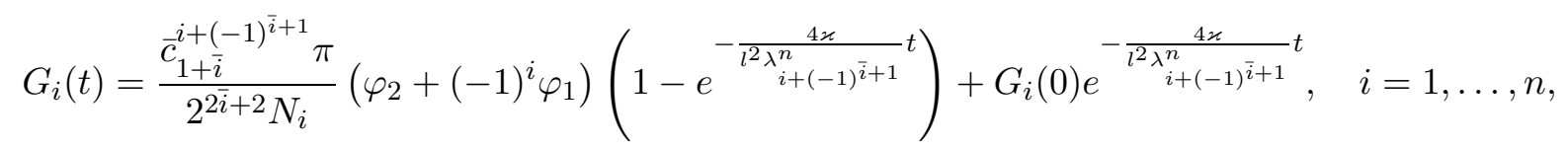

where the coefficients $G_{i}(0)$ are found from the initial condition (7) by the formula

$$
G_{i}(0)=\frac{1}{N_{i}} \int_{-1}^{1} \frac{P(x, 0) \bar{V}_{i}^{n+\bar{i}}(x)}{\sqrt{1-x^{2}}} d x .
$$

Thus, we have found the solution of the equation (6) on the interval $x \in[-1,1]$, namely

$$
P(x, t)=\sum_{i=1}^{n+2} V_{i}^{n+\bar{i}}(x)\left(\frac{\bar{c}_{1+\bar{i}}^{i+(-1)^{\bar{i}+1}} \pi}{2^{2 \bar{i}+2} N_{i}}\left(\varphi_{2}+(-1)^{i} \varphi_{1}\right)\left(1-e^{-\frac{4 \varkappa}{l^{2} \lambda^{n}} t+(-1)^{\bar{i}+1}} t\right)+G_{i}(0) e^{-\frac{4 \varkappa}{l^{2} \lambda^{n}} t+(-1)^{\bar{i}+1}} t\right) .
$$

Let us return to the variable $y$ in obtained solution and obtain the solution of the equation (2)

$$
\begin{aligned}
& p(y, t)=\sum_{i=1}^{n+2} V_{i}^{n+\bar{i}}\left(\frac{2 y-l}{l}\right) \\
& \times\left(\frac{\bar{c}_{1+\bar{i}}^{i+(-1)^{\bar{i}+1}} \pi}{2^{2 \bar{i}+2} N_{i}}\left(\varphi_{2}+(-1)^{i} \varphi_{1}\right)\left(1-e^{-\frac{4 \varkappa}{l^{2} \lambda_{i+(-1)}^{n}+1} t}\right)+G_{i}(0) e^{-\frac{4 \varkappa}{l^{2} \lambda_{i+(-1)^{n}}^{n}+1}} t\right) .
\end{aligned}
$$

\begin{tabular}{|c|c|c|c|c|c|c|c|c|c|c|c|}
\hline \multirow{2}{*}{$t$} & \multicolumn{11}{|c|}{$y$} \\
\hline & 0 & 1 & 2 & 3 & 4 & 5 & 6 & 7 & 8 & 9 & 10 \\
\hline 0 & 70.0000 & 56.0267 & 52.6886 & 55.1106 & 54.3511 & 53.7224 & 55.4532 & 54.0919 & 54.9389 & 54.6221 & 55.0000 \\
\hline 24 & 70.0000 & 60.4508 & 55.6144 & 54.4376 & 54.3707 & 54.4451 & 54.5601 & 54.6542 & 54.7525 & 54.8763 & 55.0000 \\
\hline 120 & 70.0000 & 65.3781 & 61.3767 & 58.3828 & 56.4559 & 55.4035 & 54.9364 & 54.7972 & 54.8148 & 54.8966 & 55.0000 \\
\hline 240 & 70.0000 & 66.7222 & 63.6759 & 61.0459 & 58.9397 & 57.3793 & 56.3153 & 55.6536 & 55.2837 & 55.0979 & 55.0000 \\
\hline 360 & 70.0000 & 67.3318 & 64.7914 & 62.4891 & 60.5032 & 58.8729 & 57.5973 & 56.6414 & 55.9437 & 55.4259 & 55.0000 \\
\hline 480 & 70.0000 & 67.6952 & 65.4725 & 63.4048 & 61.5476 & 59.9338 & 58.5711 & 57.4429 & 56.5106 & 55.7188 & 55.0000 \\
\hline 720 & 70.0000 & 68.1024 & 66.2438 & 64.4597 & 62.7777 & 61.2156 & 59.7792 & 58.4621 & 57.2462 & 56.1038 & 55.0000 \\
\hline
\end{tabular}

\section{Computational experiment}

The results of the solution of the equation (2) for $k=4 \cdot 10^{-12}, \mu=1.1 \cdot 10^{-6}\left(\mathrm{~m}^{2} / \mathrm{s}\right), \alpha=0.8, m=0.28$, $p_{0}=6.864655\left(\mathrm{MN} / \mathrm{m}^{2}\right), p_{1}=5.3936575\left(\mathrm{MN} / \mathrm{m}^{2}\right), \rho=998\left(\mathrm{~kg} / \mathrm{m}^{3}\right), g=9.8\left(\mathrm{~m} / \mathrm{s}^{2}\right), h_{0}=541(\mathrm{~m})$ is indicated in the tables.

Table 1. The values of the water pressure in an infinite layer of the thickness $l=10 \mathrm{~m}$ for different values of the time $t$ and the coordinate $y$ (meters) at $n=10$, the time value $t$ is indicated in hours in the table. 
Table 2. The values of the water pressure in an infinite layer of the thickness $l=10 \mathrm{~m}$ for different values of the time $t$ and the coordinate $y$ (meters) at $n=10$, the time value $t$ is indicated in hours in the table.

\begin{tabular}{|c|c|c|c|c|c|c|c|c|c|}
\hline \multirow{2}{*}{$t$} & \multicolumn{9}{|c|}{$y$} \\
\cline { 2 - 10 } & 1 & 2 & 3 & 4 & 5 & 6 & 7 & 8 & 9 \\
\hline 168 & 66.0826 & 62.5512 & 59.6844 & 57.5939 & 56.2329 & 55.4537 & 55.0777 & 54.9471 & 54.9466 \\
\hline 336 & 67.2362 & 64.6141 & 62.2544 & 60.2411 & 58.6131 & 57.3652 & 56.4553 & 55.8149 & 55.3603 \\
\hline 504 & 67.7511 & 65.5780 & 63.5482 & 61.7136 & 60.1054 & 58.7315 & 57.5771 & 56.6069 & 55.7689 \\
\hline 672 & 68.0429 & 66.1309 & 64.3045 & 62.5958 & 61.0249 & 59.5984 & 58.3087 & 57.1350 & 56.0455 \\
\hline 840 & 68.2190 & 66.4656 & 64.7646 & 63.1356 & 61.5913 & 60.1360 & 58.7652 & 57.4662 & 56.2194 \\
\hline 1008 & 68.3270 & 66.6710 & 65.0471 & 63.4676 & 61.9403 & 60.4677 & 59.0472 & 57.6710 & 56.3271 \\
\hline 1176 & 68.3934 & 66.7973 & 65.2210 & 63.6721 & 62.1552 & 60.6721 & 59.2211 & 57.7973 & 56.3935 \\
\hline 1344 & 68.4344 & 66.8752 & 65.3282 & 63.7980 & 62.2876 & 60.7980 & 59.3282 & 57.8752 & 56.4344 \\
\hline 1512 & 68.4596 & 66.9231 & 65.3942 & 63.8756 & 62.3692 & 60.8756 & 59.3942 & 57.9231 & 56.4596 \\
\hline 1680 & 68.4751 & 66.9526 & 65.4348 & 63.9233 & 62.4194 & 60.9233 & 59.4348 & 57.9526 & 56.4751 \\
\hline 1848 & 68.4847 & 66.9708 & 65.4598 & 63.9528 & 62.4504 & 60.9528 & 59.4598 & 57.9708 & 56.4847 \\
\hline 2016 & 68.4905 & 66.9820 & 65.4753 & 63.9709 & 62.4694 & 60.9709 & 59.4753 & 57.9820 & 56.4905 \\
\hline
\end{tabular}

Table 3. The values of the water pressure in an infinite layer of the thickness $l=10 \mathrm{~m}$ for different values of the time $t$ and the coordinate $y$ (meters) at $n=10$, the time value $t$ is indicated in hours in the table.

\begin{tabular}{|c|c|c|c|c|c|c|c|c|c|}
\hline \multirow{2}{*}{$t$} & \multicolumn{9}{|c|}{$y$} \\
\cline { 2 - 10 } & 1 & 2 & 3 & 4 & 5 & 6 & 7 & 8 & 9 \\
\hline 720 & 68.1024 & 66.2438 & 64.4597 & 62.7777 & 61.2156 & 59.7792 & 58.4621 & 57.2462 & 56.1038 \\
\hline 1440 & 68.4502 & 66.9053 & 65.3697 & 63.8469 & 62.3390 & 60.8469 & 59.3697 & 57.9053 & 56.4502 \\
\hline 2160 & 68.4938 & 66.9881 & 65.4837 & 63.9808 & 62.4798 & 60.9808 & 59.4837 & 57.9881 & 56.4938 \\
\hline 2880 & 68.4992 & 66.9985 & 65.4980 & 63.9976 & 62.4975 & 60.9976 & 59.4980 & 57.9985 & 56.4992 \\
\hline
\end{tabular}

\section{Solving of differential equations systems in the presence of fractional derivatives using the orthogonal method}

During the modeling many physical processes, in particular the mass transfer, it is important to consider the process history. Mathematical modeling of physical processes is usually reduced to the differential equations construction (or their systems) in partial derivatives and the formulation of the appropriate problems of mathematical physics. The models of such kind do not take into account the history of the process. Therefore, fractional (differential and integral) calculations are increasingly used for the study of such processes [7-12]. The analytical methods for solving of the problems that arise are usually constructed on the basis of Laplace operational transform [13]. The available tables of correspondence between the originals and the images or the use of contour integration don't always lead to the desired result. The use of the approximate methods of inversion can't guarantee the necessary accuracy of the original restoring. One of the effective approaches to avoid this problem is the use of the spectral methods in different bases in particular in the Laguerre polynomials basis to apply the problems of the fractional calculus.

The proposed paper has a purpose to research the method of solving of differential fractional derivatives using the spectral method in the Chebyshev-Laguerre polynomials basis.

\subsection{Fractional derivatives definition}

Several types of the fractional derivatives and integrals are introduced in the literature. The fractional derivatives in Caputo and Riemann-Liouville terms are the most used. The fractional derivative operator in Caputo terms is defined as follows [9-12]:

$$
{ }^{c} D_{\tau}^{\alpha}=\frac{{ }^{c} \partial^{\alpha}}{\partial \tau^{\alpha}} \varphi(\tau):=\frac{1}{\Gamma(m+1-\alpha)} \int_{0}^{\tau}\left(\frac{\partial^{m+1}}{\partial \xi^{m+1}} \varphi(\xi)\right) /(\tau-\xi)^{\alpha-m} d \xi
$$

Mathematical Modeling and Computing, Vol. 4, No. 2, pp. 187-196 (2017) 
where $m=[\alpha],[\cdot]$ is the integer part of real number, and in Riemann-Liouville terms

$$
D_{t}^{\alpha}=\frac{\partial^{\alpha}}{\partial t^{\alpha}} \varphi(t):=\frac{1}{\Gamma(m+1-\alpha)} \frac{\partial^{m+1}}{\partial \xi^{m+1}} \int_{0}^{t} \frac{\varphi(\xi)}{(t-\xi)^{\alpha-m}} d \xi
$$

Taking into account the given definitions we can conclude that the fractional derivatives are nothing but an integral convolution of the desired solution and another function, e.g. the power function. It is known $[2,14,15]$ that in such cases it is expedient to apply the Chebyshev-Laguerre polynomials because they are orthogonal on the semi-axis and during the expansion of the desired solution and the kernel by the given polynomials the integral convolution passes exactly to the convolution of the accordant series. Herewith, the sampling procedure which contributes a significant error into the end result in such operations is excluded.

\subsection{Formulation of the problem}

To solve many applied problems related to the nonstationary gas motion process in horizontal pipelines the linearized system of the partial differential equations that looks like the formula (18) [15] is used

$$
\left\{\begin{array}{l}
\frac{\partial \omega(x, t)}{\partial t}+\frac{\partial p(x, t)}{\partial x}+a \omega(x, t)-b p(x, t)=0 \\
\frac{\partial \omega(x, t)}{\partial x}+\frac{1}{c^{2}} \frac{\partial p(x, t)}{\partial t}=0
\end{array}\right.
$$

where $p, \omega$ are the pressure and the mass velocity of gas motion accordingly; $t$ is the time; $x$ is the movable coordinate, $x \in[0, L] ; L$ is the length of pipeline, $a=v_{1}+v_{2}, b=-\frac{1}{4}\left(v_{1}^{2}+v_{2}^{2}\right)$, and $v_{1}$ and $v_{2}$ are the limits of change of gas motion velocity, $c$ is the sound speed in gas.

It is evident that to formulate the accordant problem of mathematical physics, it is necessary to set the initial and limiting (boundary) conditions for the gas pressure and or the volumetric mass consumption which are the desired functions. The boundary conditions for the desired functions are set depending on known input data.

In Riemann-Liouville fractional derivatives terms (17), the system (18) will be written in the form

$$
\left\{\begin{array}{l}
\frac{1}{\Gamma(1-\alpha)} \frac{\partial}{\partial t} \int_{0}^{t} \frac{\omega(x, \zeta)}{(t-\zeta)^{\alpha}} d \zeta+\frac{\partial p}{\partial x}+a \omega-b p=0 \\
\frac{\partial \omega}{\partial x}+\frac{1}{c^{2}} \frac{1}{\Gamma(1-\alpha)} \frac{\partial}{\partial t} \int_{0}^{t} \frac{p(x, \zeta)}{(t-\zeta)^{\alpha}} d \zeta=0
\end{array}\right.
$$

The problem is to find the pressure distribution and the volumetric flow rate over the given domain under the given initial and boundary conditions on the desired functions (the pressure and volumetric flow rate).

\subsection{Solving of the problem}

Let us present the unknown functions $p(x, t)$ and $\omega(x, t)$ in the form of the series by the orthogonal Chrbyshev-Laguerre polynomials $L_{n}^{\lambda}(t)$, where $\lambda>-1$ is the arbitrary parameter [2]

$$
p(x, t)=t^{\lambda} \sum_{m=0}^{\infty} \frac{p_{m}(x)}{r_{m}} L_{m}^{\lambda}(t), \quad \omega(x, t)=t^{\lambda} \sum_{m=0}^{\infty} \frac{\omega_{m}(x)}{r_{m}} L_{m}^{\lambda}(t),
$$

where the coefficients $p_{m}(x), \omega_{m}(x)$ are determined by the integral relations

$$
p_{m}(x)=\int_{0}^{\infty} e^{-t} p(x, t) L_{m}^{\lambda}(t) d t \quad \text { and } \quad \omega_{m}(x)=\int_{0}^{\infty} e^{-t} \omega(x, t) L_{m}^{\lambda}(t) d t .
$$

Mathematical Modeling and Computing, Vol.4, No. 2, pp. 187-196 (2017) 
The integral equations kernel is also presented in a similar way (19)

$$
k(t)=t^{\lambda} \sum_{m=0}^{\infty} \frac{k_{m}}{r_{m}} L_{m}^{\lambda}(t) .
$$

In such presentation the series coefficients will have the form

$$
k_{m}=\int_{0}^{\infty} e^{-t} L_{m}^{\lambda}(t) k(t) d t
$$

and the normalizing multiplier $r_{m}$ is calculated by the formula

$$
r_{m}=\int_{0}^{\infty} e^{-x} L_{m}^{\lambda}(x) L_{m}^{\lambda}(x) d x=\frac{(1+\lambda)_{m}(\lambda)_{m}}{m ! m !}{ }_{3} F_{2}(-m, 1,1-\lambda ; \lambda+1,1-\lambda-m ; 1) .
$$

In our case generalized Fourier-Laguerre spectra for the function $k(t)=t^{-\alpha}$ are defined as follows

$$
k_{m}=\frac{\Gamma(m+\lambda+\alpha) \Gamma(1-\alpha)}{\Gamma(m+1) \Gamma(\lambda+\alpha)} .
$$

For the large values $m$

$$
k_{m} \approx \frac{\Gamma(1-\beta)}{\Gamma(\lambda+\beta)} m^{\lambda+\alpha-1} .
$$

The latter formula gives the opportunity to evaluate the impact of the free parameter $\lambda$ on the convergence velocity of the accordant series. However, the function representation by the series of type (20) and (22) is advantages even more so that the agreement of the choice of parameter $\lambda$ with the behavior of the function $k(t)$ accelerates the rate of the series convergence. Let us represent the functions $k(t)$ and $p(x, t)$ in the form of Fourier series by the polynomials $L_{n}^{\lambda_{k}}(t), \lambda_{k}>-1$, and $L_{n}^{\lambda_{p}}(t), \lambda_{p}>-1$, accordingly. Since [2]

$$
\int_{0}^{t}(t-\tau)^{\lambda_{k}} L_{m}^{\lambda_{k}}(t-\tau) \tau^{\lambda_{f}} L_{n}^{\lambda_{f}}(\tau) d \tau=\frac{(n+m) !}{n ! m !} B\left(\lambda_{k}+m+1, n+\lambda_{f}+1\right) t^{\lambda_{k}+\lambda_{f}+1} L_{n+m}^{\lambda_{k}+\lambda_{f}+1}(t)
$$

then the equation

$$
\frac{\partial \omega}{\partial x}+\frac{1}{c^{2}} \frac{1}{\Gamma(1-\alpha)} \frac{\partial}{\partial t} \int_{0}^{t} \frac{p(x, \zeta)}{(t-\zeta)^{\alpha}} d \zeta=0
$$

has the form

$$
\begin{aligned}
\left.\frac{\partial \omega}{\partial x}+\frac{1}{c^{2}} \frac{1}{\Gamma(1-\alpha)} \frac{\partial}{\partial t} \sum_{m=0}^{\infty} \frac{m ! k_{m}}{\Gamma(m+} \lambda_{k}+1\right) & \sum_{n=0}^{\infty} \frac{n ! p_{n}(x)}{\Gamma\left(n+\lambda_{p}+1\right)} \\
& \times \frac{(n+m) !}{n ! m !} B\left(\lambda_{k}+m+1, n+\lambda_{p}+1\right) t^{\lambda_{k}+\lambda_{p}+1} L_{n+m}^{\lambda_{k}+\lambda_{p}+1}(t)=0
\end{aligned}
$$

or

$$
\begin{aligned}
\frac{\partial \omega}{\partial x}+\frac{1}{c^{2}} \frac{1}{\Gamma(1-\alpha)} \sum_{m=0}^{\infty} \frac{m ! k_{m}}{\Gamma\left(m+\lambda_{k}+1\right)} \sum_{n=0}^{\infty} \frac{n ! p_{n}(x)}{\Gamma\left(n+\lambda_{f}+1\right)} \\
\quad \times \frac{(n+m) !}{n ! m !} B\left(\lambda_{k}+m+1, n+\lambda_{p}+1\right)\left(n+m+\lambda_{k}+\lambda_{p}+1\right)_{1} t^{\lambda_{k}+\lambda_{p}} L_{n+m}^{\lambda_{k}+\lambda_{p}}(t)=0 .
\end{aligned}
$$


If we regroup the summands in the double sum in the right side of the latter formula, we will obtain the equation

$$
\frac{\partial \omega}{\partial x}+\frac{1}{c^{2}} \frac{1}{\Gamma(1-\alpha)} t^{\lambda_{k}+\lambda_{p}} \sum_{n=0}^{\infty} d_{n}(x) L_{n+m}^{\lambda_{k}+\lambda_{p}}(t)=0
$$

In the latter formula

$$
d_{n}(x)=\sum_{m=0}^{n} k_{m} p_{n-m}(x)=\sum_{m=0}^{n} k_{n-m} p_{m}(x) .
$$

If we represent the mass consumption $\omega(x, t)$ as the series

$$
\omega(x, t)=t^{\lambda_{k}+\lambda_{p}} \sum_{n=0}^{\infty} \frac{n ! \omega_{n}(x)}{\Gamma\left(n+\lambda_{p}+1\right)} L_{n}^{\lambda_{k}+\lambda_{p}}(t),
$$

we will obtain the following recurrent system of ordinary differential equations of the unknown coefficients $\omega_{n}(x)$ and $p_{n}(x)$

$$
\frac{n !}{\Gamma\left(n+\lambda_{p}+1\right)} \frac{d \omega_{n}(x)}{d x}-\frac{1}{c^{2}} \frac{1}{\Gamma(1-\alpha)} d_{n}(x)=0 .
$$

A similar system is obtained from the first equation of the system (18)

$$
\frac{1}{\Gamma(1-\alpha)} \frac{\partial}{\partial t} \int_{0}^{t} \frac{\omega(x, \zeta)}{(t-\zeta)^{\alpha}} d \zeta+\frac{\partial p}{\partial x}+a \omega-b p=0
$$

Applying similar expansions in the latter equation, we obtain

$$
\begin{gathered}
\frac{n !}{\Gamma\left(n+\lambda_{p}+1\right)} \frac{d p_{n}(x)}{d x}+\frac{1}{\Gamma(1-\alpha)} c_{n}(x)+a \omega_{n}(x)-b p_{n}(x)=0, \\
c_{n}(x)=\sum_{m=0}^{n} k_{m} \omega_{n-m}(x)=\sum_{m=0}^{n} k_{n-m} \omega_{m}(x) .
\end{gathered}
$$

The systems (23) and (24) are recurrent relatively the unknown generalized spectra. Solving them we find $\omega_{n}(x)$ and $p_{n}(x)$ for the arbitrary values $n$ by the spectral method described above.

If we substitute the expansions (20) and (22) in the system (19) we will obtain the recurrent system of ordinary differential equations for determining of the unknown coefficients $\omega_{n}(x)$ and $p_{n}(x)$.

$$
\left\{\begin{array}{l}
\frac{1}{\Gamma(1-\alpha)} c_{n}(x)+p_{n}^{\prime}(x)+a \omega_{n}(x)-b p_{n}(x)=0 \\
\omega_{n}^{\prime}(x)+\frac{1}{c^{2}} \frac{1}{\Gamma(1-\alpha)} d_{n}(x)=0 .
\end{array}\right.
$$

The solution of the latter system we can find in the bases of the orthogonal or biorthogonal polynomials $[2,5,6,14]$.

\section{Discussion and conclusions}

The numerical values of the pressure presented in the tables 1-3 confirm authenticity of the obtained theoretical results and the efficiency of applying of the constructed biorthogonal polynomials for solving problems of the mathematical physics. The proposed algorithm is easy to automate and can be effectively used to solve other practical problems.

The proposed approach gives us an opportunity to construct the effective algorithm for solving of the differential equations or the systems of the differential equations in the presence of the fractional 
time derivative. More, if the input data is set in a discrete form the similar to the paper [2] the algorithm can be submitted in matrix form.

From the results obtained it follows that if $\lambda=-\alpha$ then the coefficients of function $k(t)=t^{-\alpha}$ are equal to zero for orders greater than one. So, for such a choice of the parameter $\lambda$ we will have the following formula

$$
d_{n}(x)=k_{0} p_{n}(x), \quad c_{n}(x)=k_{0} \omega_{n}(x) .
$$

However, as can be seen from the formula (24) such an approach to choosing of the parameter $\lambda$ allows one to accelerate the convergence of accordant Fourier-Laguerre series.

It is necessary to note that the summing of Fourier-Laguerre series is sensitive to the parameter $\lambda$. Therefore, there is a need of additional researches in summing operations of these series because Chebyshev-Laguerre polynomials have significant disadvantage that for the large $n$ their behavior is following

$$
L_{n}^{\lambda}(t)=\mathrm{O}\left(e^{t / 2} t^{-(2 \lambda+1) / 4} n^{(2 \lambda-1) / 4}\right)
$$

This property of the polynomials considerably narrows the problems class when the ChebyshevLaguerre polynomials are used because there are the computational difficulties during the series summing for the large values $t$. In practice this problem is solved by the introduction of the scaling multiplier. However, the change of the scaling multiplier requires the redefining of the problem and leads to instability in the desired function calculation. Therefore, Chebyshev-Laguerre transform is generalized as follows.

Introduce the integral transform

$$
f_{n}=\int_{0}^{\infty} t^{\nu \lambda+\nu-1} e^{-\mu t^{\nu}} L_{n}^{\lambda}\left(\mu t^{\nu}\right) f(t) d t
$$

where $n=0,1,2, \ldots, \mu>0,|\nu|<\infty, \nu \neq 0$. Then the reverse formula has the form

$$
f(t)=\sum_{n=0}^{\infty} \frac{n ! f_{n}}{\Gamma(n+\lambda+1)} L_{n}^{\lambda}\left(\mu t^{\nu}\right) .
$$

Choosing the free parameters $\mu$ and $\nu$ allows us to construct the regularizing algorithm for calculating Fourier-Laguerre coefficients $f_{n}$ and summing the corresponding orthogonal series.

[1] Prytula N. M., Pyanylo Ya.D., Prytula M. H. The underground gas storages (mathematical models and methods). Lviv, RASTR-7 (2015), (in Ukrainian).

[2] Pyanylo Ya. D. Projection-iterative methods of solving of direct and inverse problems of transport. Lviv, Spline (2011), (in Ukrainian).

[3] Pyanylo Ya. D., Vavrychuk P. H. Determination of the motion velocity of gas-water contact at the process of the underground gas storages work. Physical-Mathematical Modeling and Informational Technologies. 18, 165-172 (2013), (in Ukrainian).

[4] Lopuh N. B., Pyanylo Ya. D. Numerical model of gas filtration in porous media using fractional time derivatives. Mathematical methods in Chemistry and Biology. 2 (1), 98-104 (2014), (in Ukrainian).

[5] Pyanylo Ya. D., Sobko V.H. Constructing and researching of biorthogonal polynomials on Chebyshev polynomials basis. Applied problems of machanics and mathematics. 11, 135-141 (2013), (in Ukrainian).

[6] Pyanylo Ya., Sobko V. Researching of the spectral expansions properties at bases of orthogonal, quasiorthogonal and biorthogonal polynomials. Physical-Mathematical Modeling and Informational Tecnologies. 19, 146-156 (2014), (in Ukrainian).

[7] AhmadB., SivasundaramS. Existence of solutions for impulsive integral boundary value problems of fractional order. Nonlinear Analysis: Hybrid Systems. 4 (1), 134-141 (2010). 
[8] Ahmad B., SivasundaramS. On four-point nonlocal boundary value problems of nonlinear integrodifferential equations of fractional order. Appl. Math. Comput. 217 (2), 480-487 (2010).

[9] Kilbas A. A., Srivastava H. M., Trujillo J. J. Theory and Applications of Fractional Differential Equations. In North-Holland Mathematics Studies, vol. 204. Elsevier, Amsterdam (2006).

[10] Podlubny I. Fractional Differential Equations. Academic Press, San Diego-Boston-New York-LondonTokyo-Toronto (1999).

[11] Samko S. G., Kilbas A. A., Marichev O. I. Fractional Integrals and Derivatives. Theory and Applications. Gordon and Breach, Yverdon (1993).

[12] Vasiliev V.V., SimakL.A. Fractional calculus and approximation methods in the modeling of dynamic systems. Kiev, Scientific publication of NAS of Ukraine (2008), (in Russian).

[13] Ditkin V. A., Prudnikov A. P. Operational calculus. Moscow, High school (1975), (in Russian).

[14] Pyanylo Ya., Vasyunyk M., Vasyunyk I. Investigation of the spectral method of solving of fractional time derivatives in Laguerre polynomials basis. Physical-Mathematical Modeling and Informational Technologies. 18, 173-179 (2013), (in Ukrainian).

[15] Pyanylo Ya. Use of fractional derivatives for analysis of nonstationary gas motion in pipelines in the presense of compressor stations and outlets. Physical-Mathematical Modeling and Informational Technologies. 16, 122-132 (2012), (in Ukrainian).

\title{
Дослідження масоперенесення в складних пористих середовищах та трубопроводах за допомогою спектральних методів
}

\author{
П'янило Я., Собко В., Браташ О. \\ Центр математичного моделювання \\ Інституту прикладних проблем механіки і математики \\ ім. Я. С. Підстригача НАН України \\ вул. Д. Дудаєва, 15, 79005, Львів, Україна
}

\begin{abstract}
На основі побудованих авторами біортогональних поліномів запропоновано метод розв'язування задач математичної фізики, зокрема для знаходження розподілу тиску у воді в пластах підземних сховищ газу. Досліджено спосіб розв'язування задачі методом розділення змінних у базисі біортогональних поліномів. Розв'язок задачі знайдено у вигляді суми ряду біортогональних та квазіспектральних поліномів. Проведено порівняльний аналіз для різних значень параметрів. Вивчено вплив параметрів методів, зокрема порядку часткової суми, розрядної сітки та похибки обчислення на точність отриманого розв'язку. Результати обчислень подано у вигляді таблиць. Побудовано алгоритм дослідження процесу руху газу в трубопроводах з використанням похідних дробових порядків.
\end{abstract}

Ключові слова: спектральні методи, математична модель, розподіл тиску, ортогональні, біортогональні та квазіортогональні поліноми, диференціальні рівняння в частинних похідних та похідних дробових порядків.

2000 MSC: $12 \mathrm{E} 10$

УДК: 519.6:539.3

Mathematical Modeling and Computing, Vol.4, No.2, pp. 187-196 (2017) 